\title{
DETECTION OF ORAL CANCER AND NON CANCER FROM MICROSCOPIC BIOPSY IMAGES USING IMAGE PROCESSING TECHNIQUES
}

\author{
Ms. Maitri Joshi \\ Dept. of CSE \\ KLE Dr.M S Sheshgiri College of Engg. and Technology \\ Belagavi, Karnataka, India
}

\author{
Dr.V S Malemath \\ Dept. of CSE \\ KLE Dr. M S Sheshgiri College of Engg and Technology \\ Belagavi, Karnataka, India
}

\author{
Dr. Chetan Belaldavar \\ Dept. of Oral Pathology, \\ KAHER VK Institute of Dental Sciences, \\ Belagavi, Karnataka, India
}

\begin{abstract}
The study represents the detection of oral cancer and non cancer from microscopic biopsy images. Diagnosis of oral lesions histologically can be done through modern modalities. The various stages involved in this methodology including enhancement of the microscopic images, converting the images from RGB to $L^{*} a^{*} b$ color space, classify the colors in $L^{*} a^{*} b$ space using $k-m e a n s$ clustering, segmenting the nuclei, feature extraction and classification.
\end{abstract}

Index Terms: Image processing, clustering, colorspace, segmentation

\section{INTRODUCTION}

Cancer, one of the old ailments, understood to Egyptians and Greeks is seen among all multicellular creature. 'Malignant growth' signifies crab for it holds fast to any part it takes advantage of in a persistent way like a crab. Oral squamous cell carcinomas (OSCC) keep on predicting a poor anticipation, with an expected 5-year by and large survival of $56 \%$. Most by far of people who create squamous cell carcinoma of the upper aero digestive tract have a past filled with expending tobacco. Oral carcinogenesis is a multi-step procedure of collected hereditary harm prompting cell deregulation with disturbance in cell flagging, DNA-fix and cell cycle which are crucial to homeostasis.

Diagnosis of oral lesions histologically can be done through modern modalities. One such modality is digital pictures of microscopic slide, which are an evolution of modern day diagnosis. Concerning this digitalisation numerous new highlights can be handled by the PC. New applications are conceivable of picture examination and can be adjusted for routine use. In a normal tissue cell to cell adhesion and morphology of nuclei and cell shape does not change. But any etiologic agent can trigger changes in the oral epithelium causing cancer of the oral cavity. Oral squamous cell carcinoma (SCC) is the most common cancer of head and neck. Histologically carcinoma shows many dysplastic features, including change in the nuclei and cell morphology. Here we tried to differentiate histological pictures of normal oral tissues and oral canerous tissues using new method. As this is a pilot study, more emphasis was given to developing the software for canerous and non-cancerous tissues.

\section{RELATED WORK}

In[1], The author examines and introduces the advancement and assessment of a picture handling programming for PC assisted cell arrangement tallying. The projected programming comprises of a lot of preparing and scientific instruments which permits its utilization in a few uses of cell and cell structure tallying. The exhibition of the introduced programming in such purpose was checked by looking at the information given by visual examination, by two onlookers already aligned and under direction of two qualified authorities (Gathering 1) and by the PC program (Gathering 2). No measurable distinction was watched $(p<0.05)$ among the two gatherings.

In [2], The study executes a metadata formation of picture data. It keeps and procedures the various pictures verified by a pathologist amid his "slide seeing" and picture grouping ("perception way"). Contemporary, the auxiliary subtleties of the pathology information would be investigated. The outcomes were moved into a XML format. In light of this format, a report editorial manager and a hunt work were actualized. The statement editorial manager orders the "symptomatic way", which is the association from the picture seeing arrangement ("perception way") and the oral information grouping of the discoveries ("correspondence way").

In[3], A proposed system for robotized recognition and arrangement of malignant growth from minuscule biopsy pictures utilizing clinically huge and naturally interpretable highlights is proposed and analyzed. The different stages engaged with the proposed approach incorporate improvement of tiny pictures, division of foundation cells, highlights extraction, lastly the arrangement. A suitable and productive technique is utilized in every one of the plan ventures of the proposed system in the wake of making a near examination of generally utilized strategy in every classification.

In[4], In this study, customary malignant growth finding, pathologists inspect biopsies to make symptomatic appraisals generally dependent on cell morphology and tissue circulation. 


\section{International Journal of Engineering Applied Sciences and Technology, 2019 \\ Vol. 4, Issue 4, ISSN No. 2455-2143, Pages 264-267 \\ Published Online August 2019 in IJEAST (http://www.ijeast.com)}

Then again, computational expressive instruments engage target choices by using quantitative measures. This paper presents a methodical review of the computational strides in mechanized malignant growth conclusion dependent on histopathology. These computational advances are: 1.) picture pre-processing to decide the central regions, 2.) include taking out to evaluate the properties of these central regions, and 3.) grouping the central regions as dangerous or not or recognizing their harm levels.

\section{PROPOSED SYSTEM}

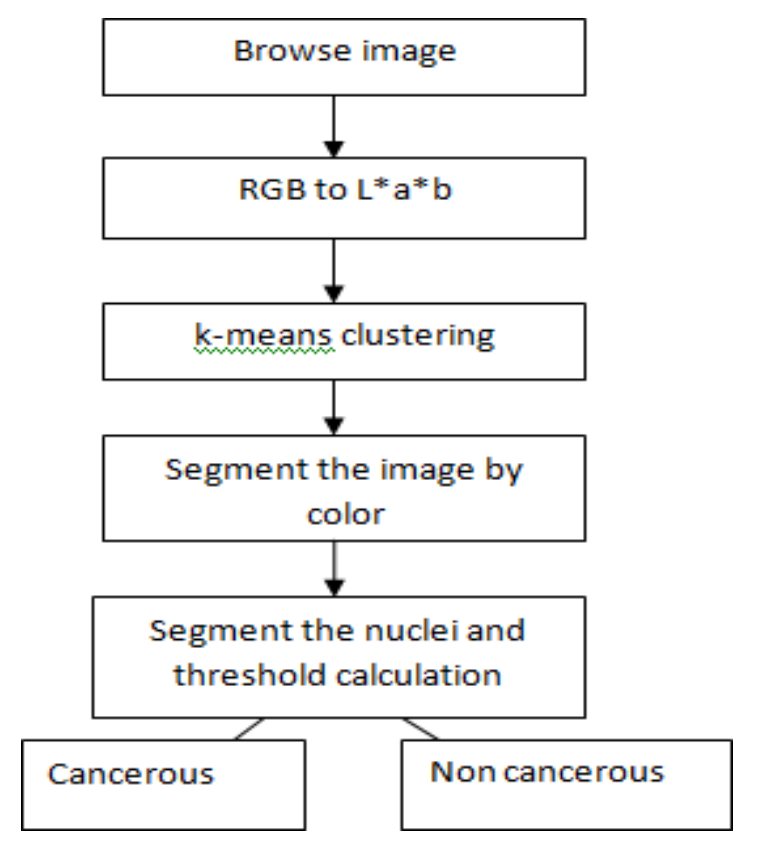

Step 1: Browse the microscopic biopsy image

Read the microscopic biopsy image which should be of the form .png, .jpg, .jpeg types. These are the images of the tissues, which are recolored with hemotoxylin and eosin. This recoloring technique causes pathologists to recognize different tissue types.

\section{Step 2: Converting the image from RGB color space to} L*a*b color space

By ignoring the variation in brightness we can identify the 3 colors like pink, blue, white. By applying the $\mathrm{L}^{*} \mathrm{a} * \mathrm{~b}$, can easily differentiate the colors from one another. It express $\mathrm{L}^{*}$ for lightness from black to white(0-100), a* for green(-) to red(+), $\mathrm{b}^{*}$ for blue(-) to yellow(+). So that color differentiation takes place easily.

Step 3: Categorize the colors in ' $a$ * $b$ ' space using k-means clustering

$\mathrm{K}$ means clustering is a type of unsupervised learning. Clustering is an approach to split gatherings of items. K- means implies grouping of every item as having an area in space. It discovers partition to such an extent that object inside each bunch are as near one another as could be allowed, and as a long way from objects in different groups as would be discreet.

It implies grouping necessitates that you demonstrate the amount of bunches to be divided and a separation metric to measure how close two items are to each another. Instead of defining groups before taking a look at the information, clustering enables you to discover and dissect the clusters that have shaped naturally. The "choosing K" segment depicts how the amount of clusters can be resolved. As the shading information exists in the ' $a * b *$ ' shading space, objects are pixels with ' $a{ }^{* \prime}$ and ' $b{ }^{* 1}$ values. Translate the data to data type single for use with imsegkmeans. Utilize imsegkmeans to group the items into 3 bunches.

\section{Step 4: segmenting the image by color}

Using pixel labels we can split the objects in image by color, which results in 3 images. The three images are separated by white, blue and pink color. Our main aim is to obtain the objects in the cluster 3.Because the cluster 3 contains the blue color objects. For the next step that is segmenting the nuclei, objects in cluster 3 image is considered.

\section{Step 5: Segmenting the Nuclei}

In the above step, the image is segmented by color, from that we have obtained the blue objects. We can distinguish the dark blue from light blue by $\mathrm{L}^{*}$ layer in the $\mathrm{L}^{*} \mathrm{a} * \mathrm{~b} *$ color space. The cell nuclei are darker blue. $\mathrm{L}^{*}$ layer contains the brightness value of each color. Obtain the brightness value of this cluster and threshold them with global threshold.

After the segmenting nuclei, count the number of blue color pixels and find the threshold. If the threshold value is between 0.04 to 0.045 it is considered as cancerous and if it is between 0.016 to 0.03 , considered as normal.

\section{RESULTS}

The planned methodology is for detection of oral cancer from microscopic biopsy images, consists of stages like convertingthe RGB color space to $\mathrm{L}^{*} \mathrm{a} * \mathrm{~b}$ color space, classification using k-means clustering and segmenting the nuclei. After the successful segmentation of the nuclei threshold value is calculated, if the value is more than 0.04 it is considered cancerous otherwise normal.

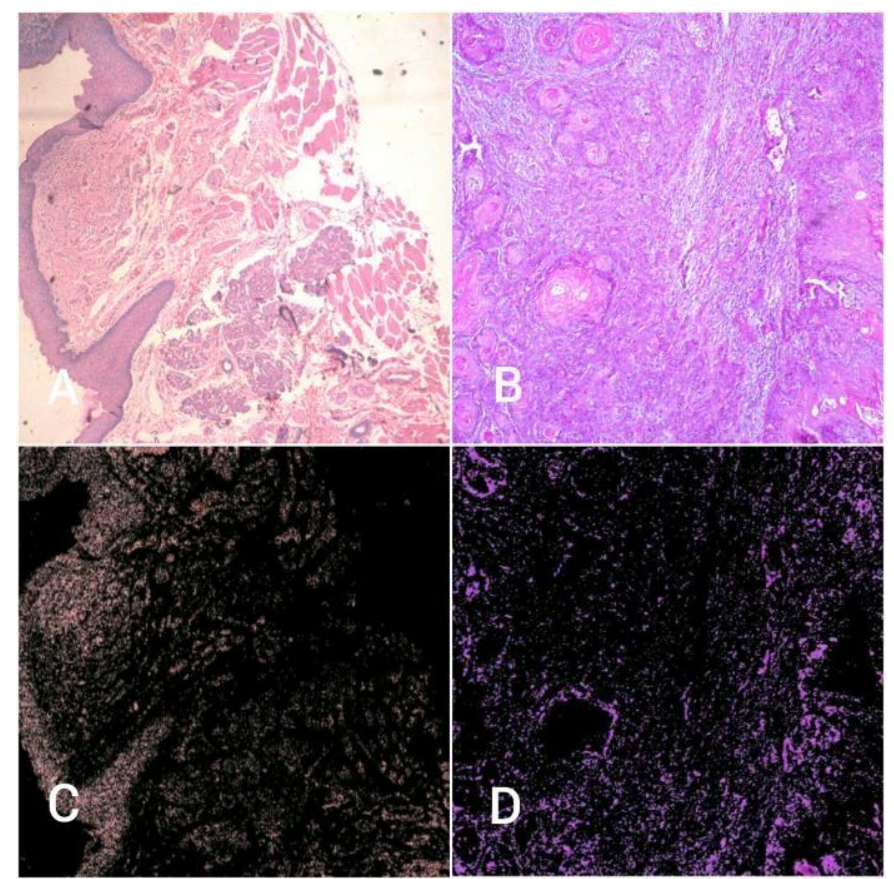

Fig4.1:A\&B indicates non cancerous and cancerous tissue tainted with hemotoxylin and eosin. $C \& D$ shows final stage output, extraction of blue nuclei 
International Journal of Engineering Applied Sciences and Technology, 2019

Vol. 4, Issue 4, ISSN No. 2455-2143, Pages 264-267

Published Online August 2019 in IJEAST (http://www.ijeast.com)

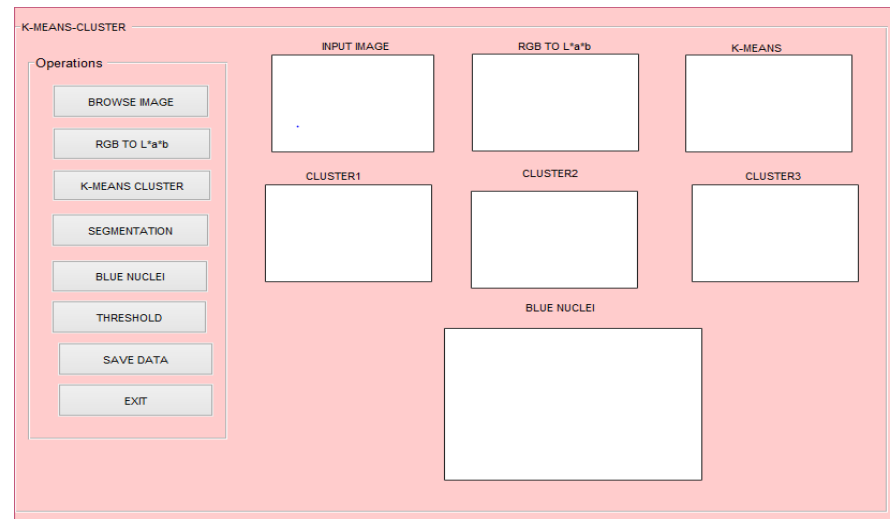

Fig4.2:Initial interface

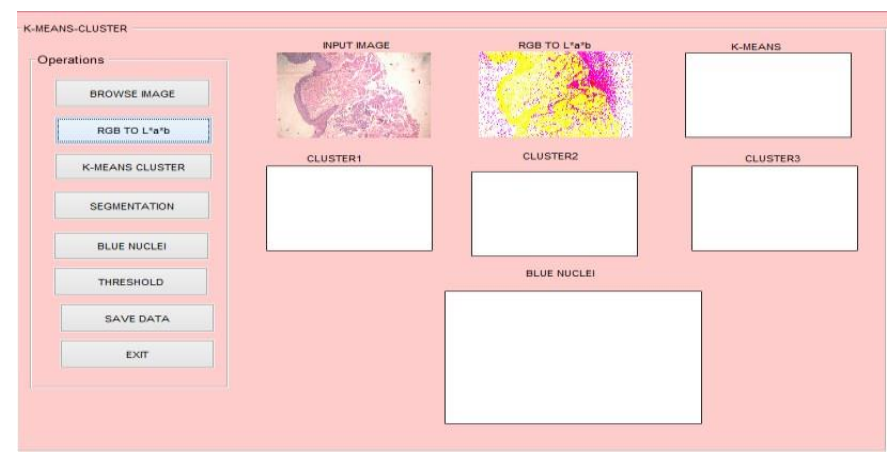

Fig4.3:Browsing the image and coversion from $R G B$ colorspace to $L * a * b$ color space

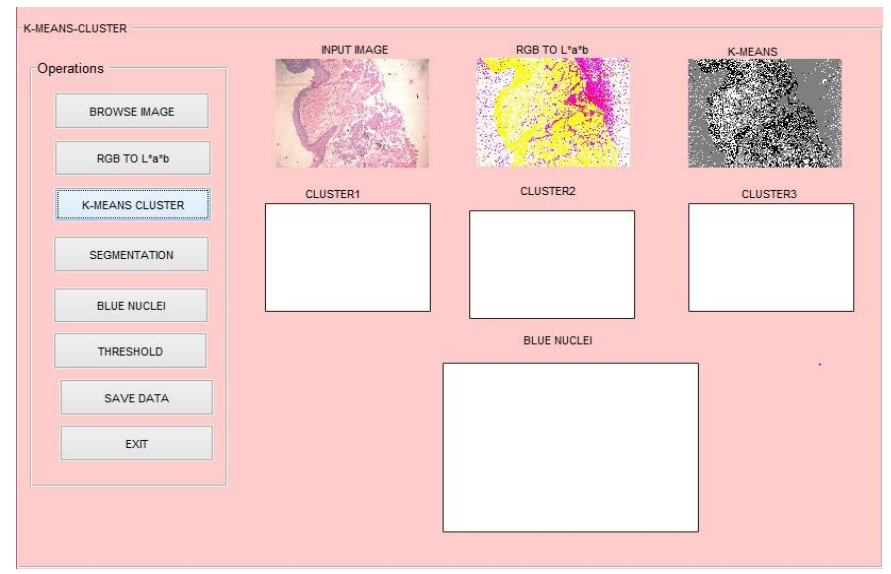

Fig4.4: classifying the colors in $a^{*} b$ space using $k$ means clustering.

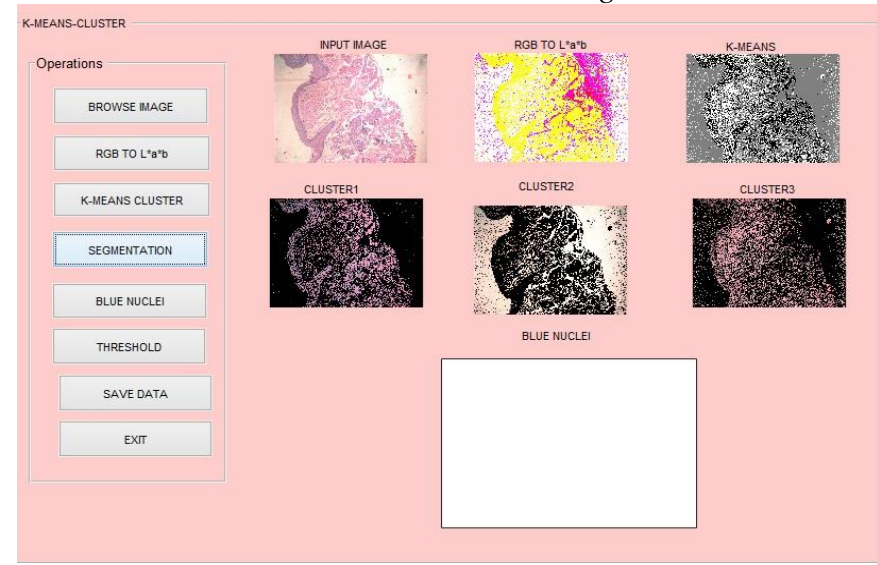

Fig4.5 : segmenting the image by color

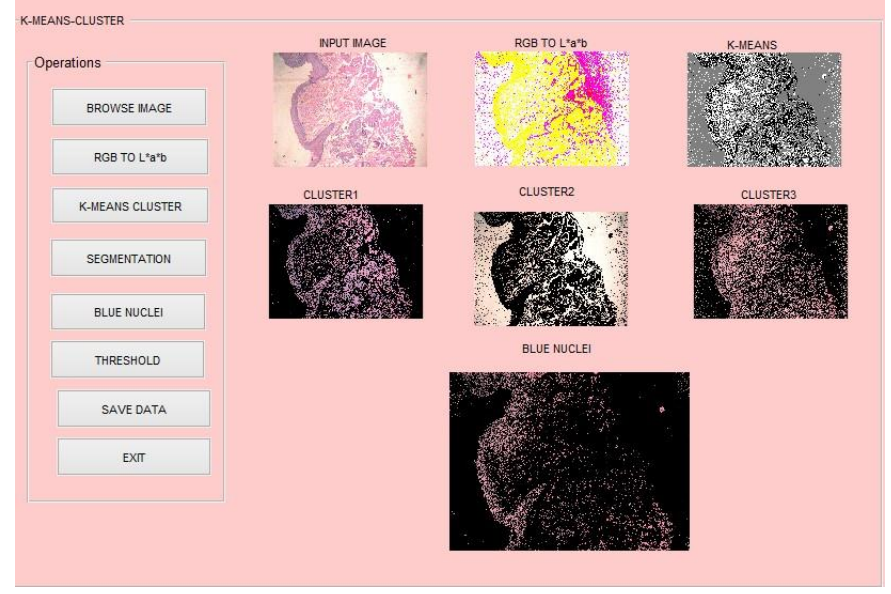

Fig4.6 :Nuclei segmentation

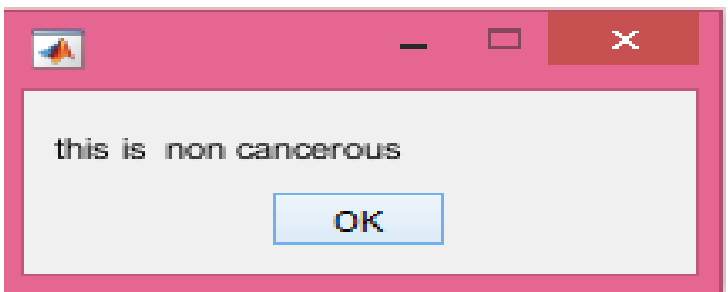

Fig4.7:Shows the type of the image

V. CONCLUSION AND FUTURE WORK

The proposed system detects the oral cancer from set of microscopic biopsy images taken from pathologist. In this approach $1 * a * b$ is used to distinguish the colors from one another. $\mathrm{K}$-means clustering is used to classify the colors. Based on threshold value it classifies the microscopic biopsy image is cancerous or normal. Furthermore it can be extended to work on different stages of cancer.

\section{REFERENCES}

[1] Alexandre A. Ferreiraa,Cristina I. Krauseb, Márcio H. Costac, Elena R.C. Riverod, Sandra B.C. Tarquínioe." An image processing software applied to oral pathology", DOI: 10.1016/j.prp.2011.02.002

[2] Schrader, Thomas et al. "The diagnostic path, a useful visualisation tool in virtual microscopy." Diagnostic pathologyvol. 140.8 Nov. 2006, doi:10.1186/1746-1596-1-40

[3]Rajesh Kumar, Rajeev Srivastava, and Subodh Srivastava, "Detection and Classification of Cancer from Microscopic Biopsy Images Using Clinically Significant and Biologically Interpretable Features". Journal of Medical Engineering, Volume 2015, Article ID 457906, doi: 10.1155/2015/457906

[4] Cigdem Demir , Bulent Yener, "Automated cancer diagnosis based on histopathological images": a systematic survey

[5] Imran Ali, Waseem A. Wani and Kishwar Saleem," Cancer Scenario in India with Future Perspectives", Cancer Therapy Vol 8, 56-70, 2011

[6]MounirSayadi,FarhatFnaiech,KarimaMrad,Khaled BenRomdhane," Automatic image segmentation of nuclear 
stained breast tissue sections using color active contour model and an improved watershed method", journal ISSN :1746-

8094,DOI:10.1016/j.bspc.2013.04.003

[7]Byun J, Verardo MR, Sumengen B, Lewis GP, Manjunath BS, Fisher SK, Automated tool for the detection of cell nuclei in digital microscopic Images":application to retinal images, Molecular Vision, vol. 12, pp. $949-960$

[8]Pal, Nikhil R. ; Pal, Sankar K. (1993) A review on image segmentation techniques Pattern Recognition, 26 (9). pp. 1277-1294. ISSN 0031-3203

[9] Tabesh, A., Teverovskiy, M., Pang, H.-Y.,Kumar, V.P,” Multifeature prostate cancer diagnosis and gleason grading of histological images, DOI: 10.1109/TMI.2007.898536

[10] C. Bergmeir, M. G. Silvente, and J. M. Benítez," Segmentation of cervical cell nuclei in high-resolution microscopic images: a new algorithm and a web-based software framework", Comput Methods Programs Biomed. 2012 Sep;107(3):497512.doi: 10.1016/j.cmpb.2011.09.017. Epub 2012 Feb 10. 